\title{
Deontología periodística sobre violencias contra las mujeres. Un estudio retrospectivo (1999-2018)
}

\section{Journalistic deontology on violence against women. A retrospective study (1999-2018)}

\author{
Aurora Edo; Belén Zurbano-Berenguer
}

Cómo citar este artículo:

Edo, Aurora; Zurbano-Berenguer, Belén (2019). “Deontología periodística sobre violencias contra las mujeres. Un estudio retrospectivo (1999-2018)". El profesional de la información, v. 28, n. 4, e280419.

https://doi.org/10.3145/epi.2019.jul.19

Artículo recibido el 09-01-2019

Aceptación definitiva: 09-04-2019

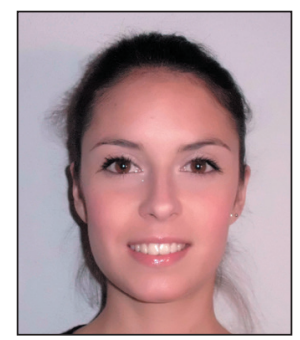

Aurora Edo $₫$
https://orcid.org/0000-0001-9718-7219
Universidad Cardenal Herrera-CEU
Departamento de Ciencias Políticas, Ética
y Sociología, Facultad de Derecho
Avda. Seminario, s/n.
46113 Moncada (Valencia), España
aurora.edo@uchceu.es

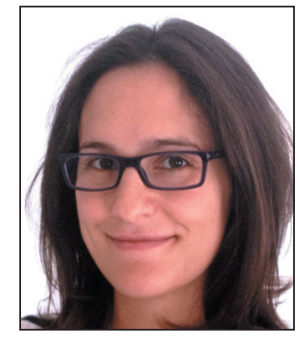

Belén Zurbano-Berenguer

https://orcid.org/0000-0002-2743-236X

Universidad de Sevilla

Facultad de Comunicación

Américo Vespucio, $\mathrm{s} / \mathrm{n}$.

41092 Sevilla, España

bzurbano@us.es

\section{Resumen}

Esta investigación aborda la producción deontológica sobre violencias contra las mujeres y medios de comunicación a partir de 58 códigos publicados entre 1999 y 2018. El objetivo principal es analizar el nivel de compromiso de los códigos con un periodismo más sensible para con el tratamiento de estas violencias. La metodología empleada se basa en el diseño de indicadores y subindicadores mediante un proceso inductivo y basado en la bibliografía científica previa. Para la medición del compromiso de cada unidad de análisis se han establecidos tres rangos de valoración: óptimo, aceptable y negativo. Entre los resultados obtenidos se destaca que el $9 \%$ de los códigos refleja un nivel de compromiso óptimo, el $53 \%$ ofrece un resultado negativo y el resto (38\%) se sitúa en el rango de aceptable. Como conclusión principal puede afirmarse que la proliferación exponencial de códigos no se corresponde con un nivel de compromiso alto de los mismos, sino con lo que parece una acción situada en la deseabilidad social y el comportamiento políticamente correcto.

\section{Palabras clave}

Violencias contra las mujeres; Deontología; Códigos; Compromiso; Medios de comunicación; Feminismo; Periodismo; España; América Latina.

\begin{abstract}
This research focuses on the study of the deontological production on violence against women (VAW) and media from 1999 to 2018, compiling 58 guidelines. Objective: To analyse the guidelines' commitment level according to a more sensitive journalism for the VAW coverage. Method: indicators have been created through an inductive process and based on the previous scientific literature. For the measurement of the commitment level three ranges of measurement have been established which are optimal, acceptable and negative. Results: only $9 \%$ of the guidelines reflect an optimal commitment level, whilst $53 \%$ offer a negative result in terms of commitment; the rest (38\%) is within the acceptable range. Conclusion: the build-up of guidelines does not correspond to a high level of commitment, but rather seems to be an action based on social desirability and politically correct behavior.
\end{abstract}

\section{Keywords}

Violence against women; Deontology; Guidelines; Commitment; Media; Feminism; Journalism; Spain; Latin America. 


\section{Introducción}

En las últimas décadas existe una preocupación creciente, tal y como lo demuestra el incremento de la producción científica sobre la materia (Zurbano-Berenguer; Cano-Orón; Liberia-Vayá, 2018), por la forma en la que los medios de comunicación transmiten y representan las violencias contra las mujeres (vvcmm) ${ }^{1}$. Esta preocupación ha derivado en una proliferación, desde finales del siglo XX, de documentos de carácter deontológico² sobre cómo abordar correcta, ética y responsablemente este problema global de derechos humanos en el periodismo.

El impacto de la labor de los medios en la configuración de la opinión pública en este ámbito específico también se ha trasladado a la investigación académica y es posible encontrar trabajos que examinan el contenido de estos manuales, su autoría institucional, el grado de implementación de las recomendaciones en la actividad periodística y, por consiguiente, su grado de efectividad. Las conclusiones generales son:

- existe un contenido altamente homogéneo en las propuestas de los códigos;

- su procedencia se caracteriza por su heterogeneidad;

- el seguimiento de las recomendaciones no alcanza el ideal ético recogido en los códigos.

Así pues, la investigación realizada viene a sumarse a los esfuerzos realizados anteriormente por comprender la praxis periodística y mejorarla; esfuerzos como los antecedentes teóricos en torno a los criterios de calidad periodística en materia de vvcmm de Aznar (2005), Bandrés (2011), Carballido-González (2007; 2009), Edo (2017), Gallego-Ayala (2003), López-Díez (2006; 2007; 2008), Martínez-Rodríguez (2010; 2011; 2012; 2013), Peris-Vidal (2016), Sutherland et al. (2015; 2016), Zurbano-Berenguer (2015a; 2015b), Zurbano-Berenguer; García-Gordillo (2017), Zurbano-Berenguer; Liberia-Vayá (2013) y Zurbano-Berenguer; Martínez-Fábregas (2011).

Este artículo se centra en el estudio de la producción deontológica sobre cómo abordar las vvcmm en los medios. Toma como referencia los contextos español y latinoamericano entre 1999 y 2018 y se basa en el estudio de 58 códigos.

En cuanto al origen y procedencia institucional de los protocolos en España se conocen únicamente los trabajos de Martínez-Rodríguez (2011; 2012) y de Zurbano-Berenguer (2015b) como investigaciones sistematizadas. Aunque ambas plantean diferentes clasificaciones, sus propuestas coinciden en señalar cuatro tipos de autoría:

- poderes públicos;

- organizaciones profesionales;

- sociedad civil;

- medios de comunicación.

En este sentido, su procedencia se caracteriza tanto por su heterogeneidad como por la cooperación entre los cuatro ámbitos señalados. Martínez-Rodríguez anota que

"Una de las particularidades más reseñable y positiva es que, pese a tratarse de normativas deontológicas que afectan a colectivos profesionales, su iniciativa procede en varios casos de la cooperación de éstos con diferentes administraciones que intentan velar por el mismo fin: acabar con la violencia ejercida contra las mujeres por todos los medios a su alcance" (Martínez-Rodríguez, 2012, p. 66).

Respecto al análisis del contenido de los códigos para establecer semejanzas y diferencias, los trabajos concluyen que existe una gran homogeneidad en las recomendaciones. Martínez-Rodríguez (2012) resume los diez rasgos comunes:

- cuáles son (o no) los términos más apropiados: violencia de género, violencia contra las mujeres, violencia machista, violencia doméstica, entre otros;

- qué es la violencia de género: problema social y estructural, un atentado contra los derechos humanos, la dignidad y la libertad de las mujeres;

- contextualización de los hechos: causas culturales y sociológicas. No caer en justificaciones tales como alcohol, drogas, celos o enfermedad mental;

- no estereotipar: las vvcmm no entienden de etnia, cultura, nivel educativo ni económico; se dan en todas las clases sociales;

- considerarlo lacra social y no sucesos aislados y fortuitos;

- no emplear recursos sensacionalistas para cubrir periodísticamente las vvcmm;

- ofrecer un seguimiento de las noticias, especialmente de aquellas donde las mujeres logran salir del infierno de la violencia;

- ofrecer información de servicio que sea útil para las mujeres víctimas;

- mantener el anonimato de la víctima y respetar la presunción de inocencia del agresor;

- utilizar fuentes expertas, no recurrir a familiares, vecindario y amistades.

Sin embargo, no se encuentran estudios que analicen el grado de compromiso de dichos protocolos. Consecuentemente se coincide con Aznar respecto a que la pluralidad de documentos acaba reflejándose en la calidad, rigurosidad y concreción de los mismos,

"así, hay documentos de gran nivel, fruto de un largo esfuerzo o de un trabajo colectivo riguroso, que aportan criterios y consideraciones realmente útiles; y los hay resultado de iniciativas menos esforzadas, cuyas aportaciones son menos relevantes" (Aznar, 2005, p. 48). 
Este trabajo comparte, pues, la hipótesis que sostienen algunas autoras (Zurbano-Berenguer, 2015b) que plantean que la proliferación de manuales se debe más a la autoexigencia de manifestar su posición pública en contra de las vvcmm y a favor de las políticas de igualdad que a la verdadera preocupación por el cómo los medios informan sobre la problemática y la influencia, cuanto menos negativa, que puedan estar ejerciendo sobre la opinión pública. Por lo tanto, los esfuerzos se quedan en el plano de lo discursivo, restando eficacia, calidad y rigurosidad a estos protocolos. En consecuencia, la profusa producción de códigos no se corresponde con un contenido éticamente más comprometido con la resolución periodística del tratamiento de las vvcmm.

\section{Objetivos e hipótesis}

El presente trabajo se plantea los siguientes objetivos:

O1. Diseñar una propuesta de indicadores que permitan medir el nivel de compromiso de los documentos deontológicos sobre el tratamiento informativo de las vvcmm.

O2. Establecer unos rangos de valoración con respecto al nivel de compromiso basados en tres estadios: compromiso negativo, compromiso aceptable, compromiso óptimo.

O3. Evaluar los códigos deontológicos existentes a partir del sistema de indicadores diseñado para conocer el nivel de compromiso expresado.

O4. Analizar la evolución temporal del grado de compromiso de los protocolos.

05. Comprobar el seguimiento individual de cada uno de los indicadores diseñados para la medición del nivel de compromiso.

O6. Detectar si existe o no correlación entre la participación de personal experto en comunicación y/o género en la elaboración de las guías y el grado de compromiso que éstas ofrecen.

Las hipótesis bajo las que se trabaja son:

H1. El nivel de compromiso puede medirse y operacionalizarse atendiendo a indicadores claramente descritos.

H2. El grado de compromiso de los materiales deontológicos varía con respecto a los contextos y tiempos de estudios.

H3. La presencia de personal experto contribuye a un mayor grado de compromiso.

\section{Muestra y método}

Se estudian 58 documentos deontológicos sobre vvcmm y medios de comunicación, publicados en España ${ }^{3}$ y en América Latina entre 1999 y 2018. La recopilación se ha llevado a cabo mediante la revisión de la bibliografía académica citada anteriormente, y profesional, como es el caso de los propios manuales que hacen referencia a otros códigos. Del mismo modo, las nuevas tecnologías han permitido, mediante el contacto directo con institutos de la mujer, asociaciones de prensa, colectivos feministas y organismos públicos, el diseño final de la muestra. Para la inclusión de los códigos se ha tomado como criterio determinante y excluyente que se abordase directamente -ya fuera en parte o en todo el documento- el binomio vvcmm y medios de comunicación.

Hay que destacar dos cuestiones:

- la muestra no es representativa, aunque haya tenido en su definición aspiraciones de exhaustividad y, por ende, los resultados no son representativos, aunque sí fieles a una realidad bien documentada;

- los documentos extraídos de la esfera latinoamericana -los más divulgados y accesibles- sirven únicamente a modo de ejemplo en otras coordenadas geo-políticas y de ningún modo se pretende representar al conjunto de países que la integran.

Los códigos objeto de análisis (en anexo 1) son 58, 43 españoles -publicados entre 1999 y $2018-$ y 15 latinoamericanos -publicados entre 2007 y 2017-.

El sistema categorial diseñado para medir el nivel de compromiso de los documentos se ha establecido mediante el método inductivo (Cardoso, 2000; Dávila-Newman, 2006; Rodríguez-Jiménez; Pérez-Jacinto, 2017). Es decir, se ha partido de la observación de la realidad -recopilación, lectura y análisis de los códigos- para detectar los rasgos comunes, presentes o ausentes, y convertirlos en indicadores de calidad y compromiso. La tabla 1 muestra la ficha de análisis empleada para tal medición. La evaluación se corresponde con un tipo de respuesta dicotómica: la respuesta positiva equivale a un punto (sí=1) mientras que la negativa o desconocida no suma (no/se desconoce=0). Así, a mayor puntuación obtenida, mayor nivel de compromiso detectado. 
Tabla 1. Ficha de análisis de medición del nivel de compromiso de los documentos deontológicos sobre violencias contra las mujeres

\begin{tabular}{|c|c|c|c|c|}
\hline \multicolumn{5}{|c|}{ Identificación } \\
\hline & Núm. referencia & Fecha & \multicolumn{2}{|l|}{ Autoría } \\
\hline \multicolumn{5}{|c|}{ Medición del compromiso. Indicadores } \\
\hline & & Sí & No & Se desconoce \\
\hline \multicolumn{5}{|c|}{ A) Justificación científica de la propuesta } \\
\hline \multicolumn{5}{|c|}{ P1. ¿Existe una investigación previa? } \\
\hline \multicolumn{5}{|c|}{ B) Aplicabilidad } \\
\hline \multicolumn{5}{|c|}{ P2. ¿Es claro, conciso y breve? } \\
\hline \multicolumn{5}{|c|}{ P3. ¿Incluye ejemplos? } \\
\hline \multicolumn{5}{|c|}{ P4. ¿Incluye nociones básicas? } \\
\hline \multicolumn{5}{|c|}{ P5. ¿Diferencia el contenido de los recursos audiovisuales? } \\
\hline \multicolumn{5}{|c|}{ C) Intencionalidad } \\
\hline \multicolumn{5}{|c|}{ P6. ¿Incluye el por qué y para qué? } \\
\hline \multicolumn{5}{|c|}{ P7. ¿Incluye estrategias de difusión e implantación entre la profesión? } \\
\hline \multicolumn{5}{|c|}{ D) Especialización y pertinencia de los autores } \\
\hline \multicolumn{5}{|c|}{$\begin{array}{l}\text { P8. ¿Hay constancia de la colaboración de profesionales de la comunicación y/o género en la } \\
\text { elaboración del documento? }\end{array}$} \\
\hline \multicolumn{5}{|c|}{$\begin{array}{l}\text { P9. ¿Hay constancia de la colaboración de investigadores de la comunicación y/o género en la } \\
\text { elaboración del documento? }\end{array}$} \\
\hline \multicolumn{2}{|c|}{ Total } & & & \\
\hline \multicolumn{2}{|l|}{ Evaluación } & \multicolumn{3}{|l|}{$\mathrm{N} / \mathrm{A} / \mathrm{O}$} \\
\hline
\end{tabular}

Del mismo modo, los trabajos de Aznar (2005) y Sutherland et al. (2016) han guiado en el proceso de diseño de dichos indicadores explicados a continuación:

\section{A) Justificación científica de la propuesta}

En primer lugar cabe plantearse si existen estudios previos que avalen científicamente las recomendaciones planteadas, es decir, ¿¿se ha desarrollado una investigación previa que sirva de base teórica para la formulación de las recomendaciones?

Con el término investigación se hace referencia, por un lado, a estudios que analicen el tratamiento mediático, en cualquier soporte, de las vvcmm o la recepción de tales informaciones por parte del público y sus posibles efectos y, por otro, a la realización de entrevistas o grupos de discusión donde se consulte a supervivientes, profesionales del ámbito de las vvcmm, periodistas expertos, etc., sobre cuál sería el tratamiento informativo adecuado. En este aspecto, el trabajo de Sutherland et al., en el que analizan once protocolos publicados en lengua inglesa, establece que

"Muchas de las recomendaciones parecen generarse a partir de la base de evidencia existente, [...]. Por ejemplo, las recomendaciones para situar los incidentes dentro de un contexto social más amplio se extraen de los hallazgos del estudio que muestran que la mayoría de los informes de los medios sobre violencia contra las mujeres se basan en eventos. Las recomendaciones menos comunes, como considerar los tipos y la ubicación de las imágenes, aunque no se basan en evidencia obtenida a partir de investigación empírica, reflejan preocupaciones más amplias sobre los posibles impactos de las prácticas de presentación de informes" (Sutherland et al., 2016, pp. 10-11).

Por investigación previa también se entiende la celebración de congresos, seminarios y jornadas donde expertos -académicos y profesionales- comparten conocimientos y debaten sobre la materia, obteniendo como resultado la elaboración de un documento deontológico fruto del proceso de debate y reflexión.

Por último, la consulta y revisión bibliográfica específica sobre el tema, como pueden ser otros códigos, es, asimismo, una tarea propia de la labor investigadora e, incluso, "un paso obligado en la investigación social en general". Así lo considera Valles, para quien la revisión de bibliografía es una técnica metodológica más para llevar a cabo estudios e investigación (Valles, 1999, p. 109).

\section{B) Aplicabilidad}

Es la facilidad y agilidad con que pueden emplearse las guías en la actividad periodística. Así, manuales muy extensos con explicaciones excesivamente detalladas de cada una de las recomendaciones se convierten en documentos poco manejables, prácticos y viables para su uso por parte de los profesionales de la comunicación, cuyo trabajo se caracte- 
riza por la premura e inmediatez. Por el contrario, manuales escuetos en demasía y formulados en términos abstractos y ambiguos (Soriano, 2004) que requieren de un sobresfuerzo por parte del periodista para su interpretación tampoco facilitan su aplicación. Sin embargo, aquellos protocolos que explican de manera clara, concreta y breve cada pauta a seguir favorecen, y mucho, su uso.

Si a dichas pautas, o aquellas que susciten mayores dudas, se añade que vayan acompañadas de ejemplos reales de malas y buenas praxis periodísticas, aumenta en consecuencia su comprensión y aplicabilidad.

Por otro lado, algunos de los manuales contienen un apartado diferenciado donde, de manera resumida:

- explican qué es -y qué no- la violencia machista, sus diferentes manifestaciones, el ciclo de la violencia;

- aportan cifras a nivel mundial, nacional y/o regional-local;

- nombran la legislación vigente en materia de vvcmm, también a diferentes niveles;

- desmienten tópicos y mitos que perpetúan dicha violencia;

- explican términos relacionados con el fenómeno -género, patriarcado, sexismo, androcentrismo, feminismo, machismo y desigualdades de género, entre otros-.

Estas nociones básicas sobre el fenómeno permiten al periodista formarse una idea más precisa de la realidad que está cubriendo y contextualizar un suceso que a priori parece aislado, en una situación atemporal donde sistemáticamente se violan los derechos fundamentales de las mujeres. De esta manera, su aproximación al problema puede estar despojada de los estereotipos y prejuicios que rodean a la violencia, a víctimas y a agresores.

Igualmente, cuando las guías diferencian entre recomendaciones propias del contenido textual y las dirigidas a los recursos audiovisuales se favorece su manejo. Existen, pues, protocolos que distinguen bajo un epígrafe destacado las pautas específicas en cuanto al uso de fotografías, vídeos y audios; mientras que otras guías introducen estas recomendaciones audiovisuales como una más dentro del conjunto. Atendiendo a ello, si al periodista le surge una duda relacionada con el uso de fotografías puede directamente consultar dicho apartado si éste está diferenciado, sin necesidad de repasar cada pauta hasta dar con la deseada, lo que agiliza el proceso de elaboración de la pieza periodística.

\section{C) Intencionalidad del documento}

La publicación de estos códigos a menudo va acompañada de una declaración de intenciones donde se explica la idea original, el proceso de elaboración y, sobre todo, se justifica el porqué y para qué, dando cuenta del grado de implicación y compromiso de la entidad que firma y edita el documento.

En cuanto a las estrategias de difusión entre la profesión, a diferencia de los indicadores anteriores, éste no ha surgido del análisis de los códigos y, por tanto, de su aparición y repetición. Son escasos los manuales que incluyen estrategias para su difusión e implantación. Y tampoco parece que las guías analizadas por Sutherland et al. incluyan tales estrategias, dejando sin responder cuestiones importantes como si los periodistas son conscientes de la existencia de recomendaciones:

"Si bien la mayoría de las pautas describen el proceso mediante el cual se desarrollaron las recomendaciones, aunque brevemente, pocas observan estrategias de implementación o difusión [...] No se proporciona información sobre cómo se puede lograr esto. La mayoría de las otras pautas carecían igualmente de información sobre la difusión a los profesionales de los medios o la industria en general" (Sutherland et al., 2016, p. 11).

Es evidente que son los profesionales del periodismo quienes deben conocer las recomendaciones para aplicarlas. Pero es imposible negar el desconocimiento que alega parte del colectivo en relación con su contenido específico y la propia desafección hacia estos manuales (Zurbano-Berenguer, 2015b), vistos en ocasiones, más como una injerencia externa en su ejercicio profesional que como

"un compromiso social de mejora de la comunicación" (Aznar, 2005, p. 33).

Sin embargo, no es la intención responsabilizar únicamente a los periodistas de la aplicación de los códigos. Las instituciones que editan los manuales deberían, asimismo, formular estrategias de difusión, tales como charlas, talleres, cursos o clases prácticas, para garantizar su implementación, así como realizar una labor de seguimiento de las recomendaciones que vendría a medir su efectividad real en la actividad diaria mediática ${ }^{4}$.

La Comisión Europea considera que la medición de efectividad de un código solo es posible si existen instrumentos de evaluación adecuados:

"Una evaluación adecuada de la efectividad y la eficiencia de un instrumento de política sólo sería posible si se dispusiera de buenos estudios de evaluación" (Comisión Europea, 2016, p. 35).

A esto cabría añadir la necesidad de unas condiciones laborales que garanticen los derechos, la independencia y la seguridad laboral de los periodistas, con el objetivo de poder aplicar libremente estas pautas deontológicas y ejercer así un periodismo más responsable, ético y humano, pues sin unas condiciones dignas, la labor de los profesionales queda excesivamente expuesta, redundando en un detrimento de su beneficio social. 


\section{D) Capacitación, grado de especialización y pertinencia de los autores}

Por último, es importante tener en cuenta la formación y especialización de quienes asesoran en la elaboración y redacción de los manuales. Se entiende por expertos tanto a los profesionales de los medios especialistas en comunicación y género -o de manera más específica, en el tratamiento informativo de las vvcmm- como a los académicos e investigadores también especialistas en estos temas.

La bibliografía especializada coincide en señalar la falta de especialización del colectivo de periodistas encargado de cubrir las informaciones sobre vvcmm como una de las principales causas del incorrecto, sensacionalista y, en ocasiones, irresponsable tratamiento del fenómeno. Zurbano-Berenguer y Martínez-Fábregas concluyen que

"quizá la carencia actual se fundamente en una falta de formación profesional específica, en un conocimiento especializado, profundo, de por qué ciertas prácticas han de dejarse de lado a la hora de abordar la violencia de género en los medios de comunicación" (Zurbano-Berenguer; Martínez-Fábregas, 2011, p. 2144).

Asimismo, los propios manuales recogen como una recomendación más la necesidad de formación tanto de los periodistas como de sus cargos superiores:

"Recomendamos cursos de especialización en igualdad y violencia de género a la dirección y a los mandos con poder de decisión en todos los medios de comunicación, además de a la plantilla normal de la redacción" (La marea, 2015).

Pero si es deseable que los periodistas estén formados en el tema, todavía es más importante y exigible que aquellos que recomiendan cómo tratar mediáticamente las vvcmm sean especialistas tanto por su conocimiento teórico como práctico.

Para la evaluación, de tipo proporcional, de cada documento se han establecido tres rangos diferenciados: sobre una escala de nueve puntos -correspondientes a los nueve criterios de la tabla 1-, se considerará que el documento ofrece un grado de compromiso:

- negativo (N) cuando no se alcancen los cinco puntos;

- aceptable (A) cuando el documento obtenga entre cinco y seis puntos;

- óptimo (O) cuando el documento cumpla siete o más criterios.

\section{Resultados}

Los resultados obtenidos contribuyen a comprobar algunas de las hipótesis planteadas, así como a reforzar la idea de seguir trabajando para justificar otras. Las tablas 2 y 3, muestran un resumen de los principales hallazgos obtenidos.

Tabla 2. Resumen de los hallazgos: objetivos

\begin{tabular}{|l|l|}
\hline Objetivo 1 & $\begin{array}{l}\text { Se ha diseñado un sistema categorial basado en cuatro indicadores y nueve subindicadores convertidos en preguntas cuya } \\
\text { respuesta es de tipo dicotómica (sí/no), siendo } 9 \text { la máxima puntuación posible y } 0 \text { la mínima. }\end{array}$ \\
\hline Objetivo 2 & $\begin{array}{l}\text { Se han establecido tres rangos de valoración diferenciados atendiendo a las puntuaciones posibles. Una valoración denomi- } \\
\text { nada "negativa" es aquella cuya puntuación numérica oscila entre } 0 \text { y } 4 \text { puntos, una "aceptable", aquella comprendida entre } 5 \\
\text { y 6, y una "óptima", la que se encuentra entre } 7 \text { y } 9 \text { puntos. }\end{array}$ \\
\hline Objetivo 3 & $\begin{array}{l}\text { El instrumento de medición del compromiso ha sido testado en } 58 \text { códigos deontológicos dando por resultado un proceso } \\
\text { posible, sencillo y con resultados empíricos comprobables a partir de un proceso de fácil replicabilidad científica. La aplica- } \\
\text { ción de la ficha de análisis ha permitido conocer el grado de compromiso de los materiales de la muestra. }\end{array}$ \\
\hline Objetivo 4 & $\begin{array}{l}\text { Las mediciones realizadas en los contextos español y latinoamericano permiten observar un comportamiento errático en } \\
\text { cuanto a la evolución del compromiso de los códigos. No se demuestra que con el paso del tiempo exista un mayor compro- } \\
\text { miso de los mismos, si bien parece existir una ligera tendencia positiva mayor en el contexto latinoamericano. }\end{array}$ \\
\hline Objetivo 5 & $\begin{array}{l}\text { La evaluación ha detectado que las preguntas respondidas afirmativamente en un mayor número de casos son las relativas } \\
\text { a la claridad, concisión y brevedad del documento y a la presencia de los porqués y paraqués (2 y } 6 \text { respectivamente). El ítem } \\
\text { menos recogido en los manuales son las estrategias de aplicación práctica en la profesión (pregunta 7). }\end{array}$ \\
\hline Objetivo 6 & $\begin{array}{l}\text { Del análisis relacional entre el grado de compromiso de los códigos y la participación de expertos se comprueba que cuando } \\
\text { en los manuales participan profesionales e investigadores, el 80\% de ellos presenta niveles de compromiso aceptable y } \\
\text { óptimo; y cuando no ha habido o se desconoce dicha participación, el 100\% suspende el grado de compromiso. }\end{array}$ \\
\hline
\end{tabular}

Tabla 3. Resumen de los hallazgos: hipótesis

\begin{tabular}{|l|l|}
\hline Hipótesis 1 & $\begin{array}{l}\text { Se demuestra que una operacionalización del concepto "compromiso deontológico" es posible a través del diseño de indica- } \\
\text { dores cualitativos y que la herramienta resultante aporta evidencias de interés sobre los materiales objeto de estudio. }\end{array}$ \\
\hline Hipótesis 2 & $\begin{array}{l}\text { El grado de compromiso de los protocolos es irregular porque no sigue un patrón temporal tendente al alza ni se detectan } \\
\text { diferencias importantes entre los contextos geográficos analizados. }\end{array}$ \\
\hline Hipótesis 3 & $\begin{array}{l}\text { Se confirma que la participación de personas expertas es un elemento presente en aquellos documentos con puntuaciones } \\
\text { más altas. }\end{array}$ \\
\hline
\end{tabular}

En relación con los objetivos 1 y 2, relativos al diseño instrumental y a la valoración de los documentos, ha de ponerse de manifiesto que el diseño del sistema y el testeo del mismo en la muestra han dado resultados fiables, comprobables y coherentes. 
Respecto al objetivo 3, y tras la aplicación de la ficha de medición (tabla 1), el gráfico 1 muestra que 31 de las guías (53\%) españolas y latinoamericanas ofrecen una puntuación negativa; mientras que sólo 5 (9\%) alcanzan un óptimo grado de compromiso. El resto, 22 (38\%), cumple un mínimo de cinco puntos situándose en un rango de aceptable.

De manera diferenciada (gráficos 2 y 3), 25 de las guías españolas (58\%) no superan los cinco puntos. 15 de ellas (35\%) se sitúan en el rango de aceptable y sólo 3 (7\%) alcanzan los siete puntos o más. Por su parte, el número de manuales latinoamericanos que ofrecen niveles aceptable y negativo es prácticamente el mismo -7 (47\%) y $6(40 \%)$, respectivamente-; mientras que sólo 2 (13\%) ofrecen un grado óptimo.

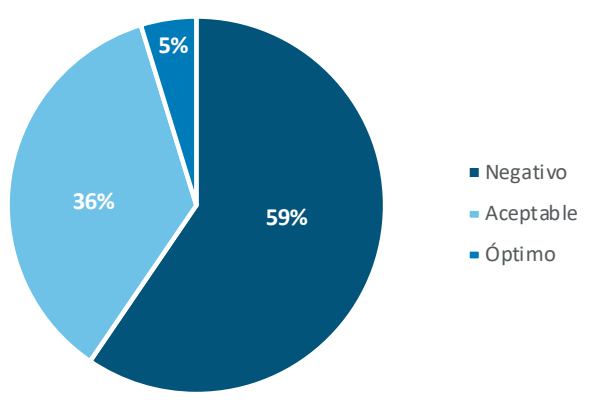

Gráfico 2. Análisis del nivel de compromiso de los documentos deontológicos españoles sobre violencias contra las mujeres

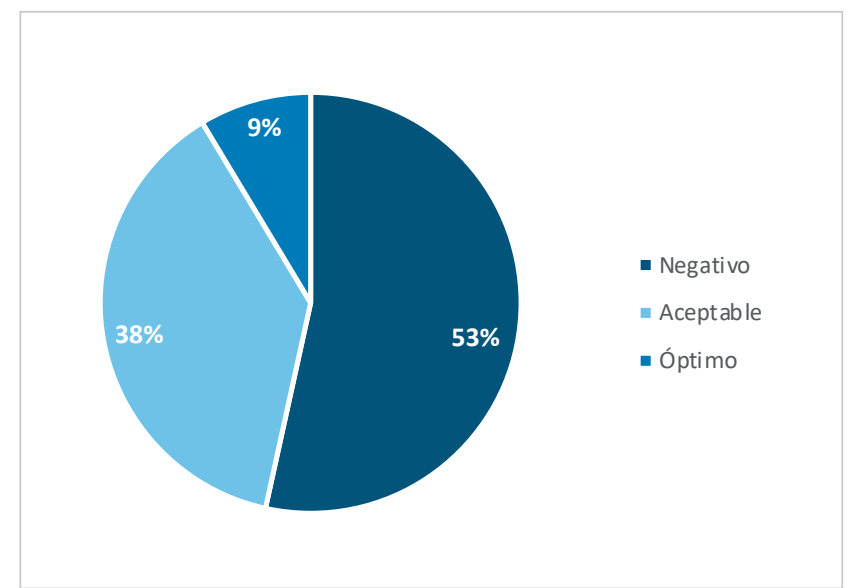

Gráfico 1. Nivel de compromiso de los documentos deontológicos españoles y latinoamericanos sobre violencias contra las mujeres

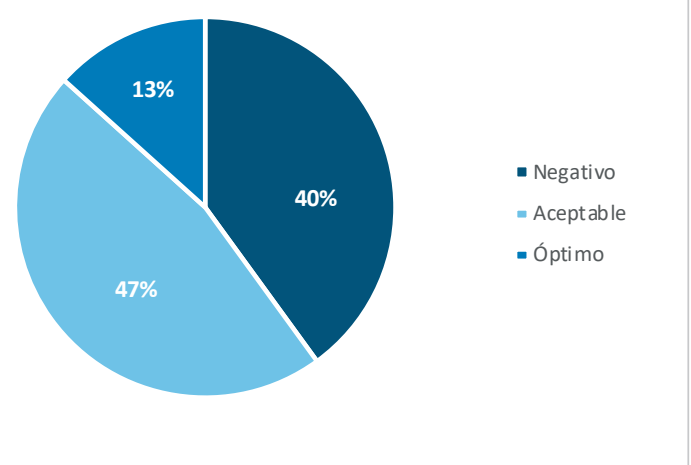

Gráfico 3. Análisis del nivel de compromiso de los documentos deontológicos latinoamericanos sobre violencias contra las mujeres

En cuanto al objetivo 4 -analizar la evolución temporal del grado de compromiso de los protocolos-, se observa que tanto los manuales españoles como latinoamericanos (gráficos 4 y 5) no mantienen una tendencia lineal al alza conforme se avanza en el tiempo, más bien su evolución es errática. En los gráficos 4 y 5 se puede apreciar el número de documentos publicados en los períodos abarcados -España, 1999-2018; América Latina, 2007-2017-y el nivel de compromiso alcanzado por cada uno de ellos.

En el gráfico 6 se muestran los resultados respecto al objetivo 5, y es destacable que la pregunta que más respuestas afirmativas ha recibido es la número seis - ¿Incluye el porqué y para qué?-. El 83\% de los documentos, españoles y latinoamericanos, contiene una explicación de cuál es la intención, los objetivos perseguidos o la utilidad de su publicación. Es decir, en la mayor parte de las guías la inclusión de estos valores conceptuales está comúnmente asentada y se considera generalmente esencial. Seguidamente, las preguntas $2-\dot{\mathrm{E} s}$ claro, breve y conciso? - y 8 - ¿Hay constancia de la colaboración de profesionales de la comunicación en la elaboración del documento?acumulan, respectivamente, más respuestas afirmativas, pues el 79\% de las recomendaciones son claras y concisas y el $69 \%$ ha contado con profesionales de la comunicación para su redacción.

Más de la mitad de las guías (57\%) cuenta con una investigación previa que justifica científicamente la propuesta deontológica (P1) y el 50\% contiene nociones básicas sobre vvcmm (P4). Mientras, en el $41 \%$ de

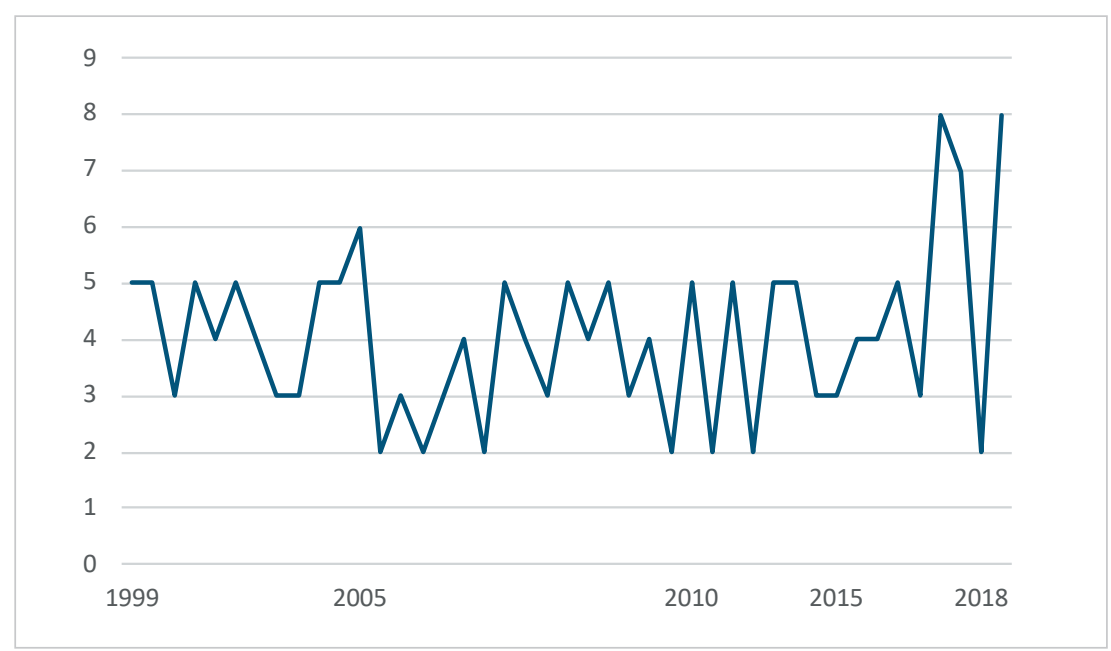

Gráfico 4. Evolución del nivel de compromiso de los documentos deontológicos españoles sobre violencias contra las mujeres 
los protocolos ha participado personal investigador en su elaboración (P9).

En los últimos puestos se encuentran las preguntas 5,3 y 7 al reunir más respuestas negativas respectivamente: el $22 \%$ de los códigos españoles y latinoamericanos diferencia las recomendaciones de contenido textual de las dirigidas a los recursos audiovisuales (P5); el $17 \%$ recoge ejemplos de buenas y malas praxis (P3) y sólo el $12 \%$ contempla medidas de difusión e implantación entre la profesión periodística (P7).

En lo que corresponde a especialización y pertinencia de los autores de los códigos, el $69 \%$ de los documentos ha contado con la participación de profesionales de la comunicación para su elaboración, mientras que el $41 \%$ ha sido elaborado, o ha recibido el asesoramiento directo, de investigadores del ámbito de la comunicación y/o del género. De manera más específica, en 15 de las guías (26\%) han participado tanto profesionales como investigadores y en 9 de ellas $(15 \%)$ se desconoce si se ha contado o no con personal experto.

En la tabla 4 se indica para cada documento -ref. 1, 2, 3 y así sucesivamente- la participación -marcada con una " $x$ "- de profesionales (P8) y de investigadores (P9) en la elaboración de cada uno de ellos. Con esto se pretende:

- mostrar de forma visual el grado de

plimiento de estos dos indicadores de compromiso de los códigos, resultando pocos los documentos en los que coincide la participación conjunta de profesionales y personal académico;

- mostrar el nivel de compromiso alcanzado (NC) por cada protocolo.

Tabla 4. Relación entre el nivel de compromiso (NC) de los documentos deontológicos sobre violencias contra las mujeres y la participación de personas expertas en su elaboración

\begin{tabular}{|c|c|c|c|c|c|c|c|c|c|c|c|c|c|c|c|c|c|c|c|}
\hline Ref. & P8 & P9 & NC & Ref. & P8 & P9 & NC & Ref. & P8 & P9 & NC & Ref. & P8 & P9 & NC & Ref. & P8 & P9 & NC \\
\hline 1 & & $x$ & 5 & 13 & $x$ & & 2 & 25 & $x$ & & 3 & 37 & $x$ & $x$ & 4 & 49 & $x$ & $x$ & 6 \\
\hline 2 & & $x$ & 5 & 14 & & & 3 & 26 & $x$ & & 4 & 38 & $x$ & $x$ & 5 & 50 & $x$ & & 5 \\
\hline 3 & $x$ & & 3 & 15 & & & 2 & 27 & $x$ & & 2 & 39 & $x$ & & 3 & 51 & $x$ & & 5 \\
\hline 4 & $x$ & $x$ & 5 & 16 & & & 3 & 28 & $x$ & $x$ & 5 & 40 & $x$ & $x$ & 8 & 52 & & & 4 \\
\hline 5 & $x$ & & 4 & 17 & $x$ & & 4 & 29 & & & 2 & 41 & $x$ & $x$ & 7 & 53 & & & 4 \\
\hline 6 & & $x$ & 5 & 18 & $x$ & & 2 & 30 & $x$ & $x$ & 5 & 42 & $x$ & & 2 & 54 & & & 4 \\
\hline 7 & $x$ & & 4 & 19 & $x$ & $x$ & 5 & 31 & $x$ & & 2 & 43 & $x$ & & 8 & 55 & & $x$ & 3 \\
\hline 8 & & & 3 & 20 & & $x$ & 4 & 32 & & $x$ & 5 & 44 & & $x$ & 5 & 56 & $x$ & & 7 \\
\hline 9 & $x$ & & 3 & 21 & $x$ & $x$ & 3 & 33 & $x$ & & 5 & 45 & $x$ & & 4 & 57 & $x$ & & 6 \\
\hline 10 & & $x$ & 5 & 22 & $x$ & $x$ & 5 & 34 & $x$ & & 3 & 46 & $x$ & & 4 & 58 & $x$ & & 6 \\
\hline 11 & $x$ & $x$ & 5 & 23 & & & 4 & 35 & & $x$ & 3 & 47 & $x$ & & 5 & & & & \\
\hline 12 & $x$ & & 6 & 24 & $x$ & $x$ & 5 & 36 & $x$ & $x$ & 4 & 48 & $x$ & $x$ & 7 & & & & \\
\hline
\end{tabular}

Negrita y verde: protocolos en cuya elaboración han participado tanto profesionales como investigadores.

Cursiva y rojo: protocolos que no han contado con personal experto. 
En respuesta al objetivo 6 -existe, o no, una correspondencia entre la especialización y pertinencia de autores y el nivel de compromiso de los documentos-, se comprueba que de los 15 protocolos en cuya elaboración han participado tanto profesionales como investigadores -destacados en negrita y verde-, 9 (60\%) ofrecen un grado de compromiso aceptable, 3 (20\%), óptimo y $3(20 \%)$, negativo. Mientras que las pautas que no han contado, o se desconoce, con personal experto -señaladas en cursiva y rojo-, presentan todas ellas una puntuación inferior a cinco puntos, siendo su grado de compromiso negativo.

Del resto de códigos en los que han participado o bien profesionales de la comunicación o bien investigadores de la comunicación y/o el género, el 56\% se sitúa en el rango negativo, el 38\%, en el aceptable y el 2\%, en el óptimo. En resumen, cuando en los manuales participan profesionales e investigadores, el $80 \%$ de ellos presenta niveles de compromiso aceptable y óptimo; cuando sólo participa uno de los dos grupos, el $44 \%$ ofrece un nivel de compromiso aceptable y cuando no ha habido o se desconoce dicha participación, el 100\% suspende el nivel de compromiso.

\section{Conclusiones}

Los resultados obtenidos confirman la hipótesis principal del estudio relativa a que la proliferación de códigos no se corresponde con el aumento del nivel de compromiso de los mismos. En general, los códigos deontológicos sobre cómo deben los medios de comunicación abordar el problema social y democrático de las vvcmm presentan evaluaciones negativas sobre su nivel de compromiso como viene poniéndose de manifiesto. Más del $50 \%$ de los protocolos analizados presenta evaluaciones negativas y sólo el $9 \%$ cumple una puntuación "óptima”. Hay que precisar que ningún manual responde positivamente a todos los indicadores de compromiso descritos.

Resulta imprescindible plantear que ya anteriormente otros han tratado de responder al objetivo general de esta investigación -mejorar la práctica profesional para el beneficio social- desde el trabajo con los profesionales. Los resultados obtenidos por ejemplo en Zurbano-Berenguer (2015b) mediante entrevistas cualitativas con periodistas encargados de estas noticias, jefes de sección y directores de medios han revelado, entre otras cuestiones, que no existe un conocimiento generalizado de estos códigos ni un debate sobre los mismos en las redacciones y, además, que los profesionales que tienen constancia de su existencia no se sienten comprometidos con ellos por diversas razones siendo la fundamental que no han participado en su elaboración. Anteriormente, Fernández-Arribas y Noblejas (2010) también plantearon aspectos relativos a la deontología y la sensibilidad con las vvcmm a profesionales del periodismo. Para casi la mitad de las personas encuestadas -casi 1.000 periodistas y 1.200 ciudadanos- los medios de comunicación deben servir para prevenir la violencia contra la mujer en las relaciones de pareja. Asimismo, en el trabajo se revelaron algunos elementos ciertamente interesantes como que el $47,06 \%$ de los periodistas encuestados consideraba más noticiosa una agresión de una mujer a un hombre que una sentencia condenatoria a un agresor de mujeres $(45,15 \%)$ o que una agresión de un hombre a una mujer (7,79\%).

En el trabajo aquí desarrollado se plantea el grado de compromiso de los códigos en sí como un eje determinante para explicar el funcionamiento deontológico del periodismo actual en vvcmm y con la pretensión de sumar una nueva arista en el abordaje de este problema.

En España los materiales con una mayor puntuación obtenida son los elaborados por La marea y Oxfam Intermón (2017) y la Unió de Periodistes Valencians (2018), cumpliendo ocho de los nueve indicadores en ambos casos. Siguiendo a éstos, se sitúa el editado por la Junta de Castilla y León (2017) atendiendo satisfactoriamente a siete de los items descritos. Se podría deducir que los tres, publicados entre 2017 y 2018, han podido basarse en el bagaje deontológico que le precede, así como en los trabajos de investigación cada vez más numerosos en este tema para elaborar unos manuales fundamentados en investigaciones previas, de fácil aplicación y que han contado con el asesoramiento directo de personas expertas en comunicación y/o género. Sin embargo, el factor temporal (gráfico 4) no es determinante en el resto de documentos, es decir, no se detecta una progresión uniforme en aumento en el nivel de compromiso conforme se avanza en el tiempo. En el lado opuesto, siete códigos responden positivamente a sólo dos criterios, siendo todos ellos publicados entre 2005 y 2018, lo que viene a confirmar la inexistencia de un patrón lineal en la evolución del nivel de compromiso.

Respecto a Latinoamérica, son los documentos de Amnistía Internacional Argentina (2009) y del Ministerio de Comunicación de Bolivia (2015) los que alcanzan el grado de compromiso más alto. En este contexto es reseñable que ninguno de los materiales ofrece niveles de compromiso tan bajos como algunos de los españoles, obteniendo sólo uno de ellos un mínimo de tres puntos. Pero, de nuevo, la evolución del nivel de compromiso no sigue una tendencia clara positiva.

Un elemento interesante que se extrae de la evaluación deontológica es el mínimo común denominador de los manuales, pues tanto en el caso español como latinoamericano, los códigos presentan de forma mayoritaria elementos explicativos del porqué y para qué, intentando dar sentido al propio documento. No puede negarse que sin otras estrategias parejas la mera exposición de intenciones -denunciar, sensibilizar, contribuir a erradicar-va a quedarse, precisamente, en una declaración de honorables intenciones sin garantías mínimas de aplicabilidad real y, por ende, de beneficio social.

De igual forma destaca que la mayoría de los códigos analizados (60\%) son fruto de una investigación previa. Esto evidencia una progresiva preocupación social tangible a partir de los procesos de reflexión, discusión e investigación que sustentan los documentos, alejándolos del carácter puramente arbitrario y subjetivo de los primeros documentos, de índole más militante que profesional. 
Para finalizar, es relevante atender a la confirmación de que la participación de personas expertas incide directamente en el nivel de compromiso de los códigos, ya que la correlación entre la participación de éstas y el grado de compromiso alcanzado por las guías es clara: su participación implica que los protocolos ofrezcan un mayor nivel de compromiso, profundización y rigurosidad, y a la inversa en el conjunto de la muestra en general.

\section{Discusión y retos de futuro}

Los medios de comunicación social operan hoy desde un rol preventivo en cuanto a las vvcmm (Simons; Khan, 2018). Así se torna necesaria y urgente una reflexión sobre cómo éstos están funcionando y sobre qué bases deontológicas orientan, al menos desde un plano teórico, su actuación.

Sobre el tratamiento pasado, actual y deseable de los medios respecto a estas violencias se han referenciado los principales estudios en la introducción de este trabajo. Sobre la cuestión del estado actual de la deontología periodístico-comunicativa al respecto también existen precedentes (Peris-Vidal, 2016; Zurbano-Berenguer, 2015b; Martínez-Rodríguez, 2011; Bandrés, 2011) que deben ponerse en relación con los hallazgos encontrados. Si los estudios actuales han permitido conocer cuáles son los valores éticos que subyacen a las recomendaciones, este trabajo ha puesto de manifiesto cuál es el grado de compromiso de estos materiales profesionales.

Resulta fundamental conocer el compromiso, dado que investigaciones anteriores han alertado de la ineficacia aparente de estos códigos. Algunos autores identifican que son dos las principales causas del no seguimiento de estas guías por parte de los periodistas:

- el rechazo por considerarlos una injerencia a su criterio profesional (Aznar, 2005);

- la falta de conocimiento de los mismos (Zurbano-Berenguer, 2015b).

Sobre las características, valores y desarrollos históricos del periodismo que hace a los profesionales acreedores de este celo profesional, podría investigarse aún desde el marco de la sociología de las profesiones, sobre todo para romper esa delicada barrera entre la autonomía y la -necesaria- especialización. Pero lo que interesa resaltar aquí es que si los materiales deontológicos continúan elaborándose sin garantías de compromiso real y no sólo desde el deseo del compromiso público o las actuaciones políticamente correctas (Zurbano-Berenguer, 2015b) los profesionales seguirán sin acceso real a los medios que pueden contribuir a mejorar el tratamiento público de las vvcmm y así coadyuvar a su erradicación.

Es cierto que la mera publicación de manuales refleja cierto "esfuerzo de sensibilización" (López-Díez, 2007, p. 41) por parte de las entidades y contribuye a "crear una cultura ética de los medios de comunicación" (Aznar, 2005, p. 48), pero si no se contemplan medidas o estrategias para dar a conocer el contenido de las guías, un indicador más de compromiso de entre los descritos, su utilidad quedará en el plano de lo teórico - de las buenas intenciones- pero no en el real y efectivo.

Además, aquellas guías que sí diseñan estas estrategias de difusión y de aplicación profesional muestran de forma diferente el grado de implicación de las entidades promotoras. Así pues, se desarrollan medidas que se limitan a hacer llegar a las redacciones de los medios sus guías -p. ej. Instituto Balear de la Mujer, 2003-mientras que otras, más sofisticadas, programan la realización de talleres -p. ej. FAPE 2005-, cursos online -p. ej. Junta de Castilla y León, 2017- o la creación de grupos de trabajo que realizan un seguimiento continuo de la implementación de los protocolos en las informaciones -p. ej. Ministerio de Comunicación de Bolivia, 2015-.

La amplia proliferación de materiales y su disparidad en el nivel de compromiso hace que las autoras de este trabajo se planteen su eficacia y su utilidad no sólo periodística sino también social. ¿Para qué sirven códigos que no se siguen y que no se siguen porque no se conocen o no se sabe cómo implementar? -ya se ha advertido de que la hipótesis de que no se quiera expresamente trabajar con ellos es materia de reflexiones paralelas-. El futuro de la investigación en el área deberá plantearse nuevamente el trabajo con los profesionales para analizar qué obstáculos median entre la existencia de unos consensos sólidos, una proliferación documental importante que recoge dichos consensos y una aún defectuosa puesta en práctica de los mismos.

El grado insostenible de violencias y las evidencias científicas acerca de cómo los medios de comunicación social contribuyen a su erradicación instan a la movilización epistémica y social. En la actualidad, las reflexiones sobre políticas y prácticas comunicativas se vuelven un reto no sólo académico sino también democrático.

\section{Notas}

1. Por violencias contra las mujeres (en adelante, vvcmm -en minúscula, siguiendo las normas editoriales-) se entiende:

"los actos y amenazas de tales actos, perpetrados en la vida pública y en la privada, que supongan peligro para la vida, la integridad, el cuerpo y la dignidad de las mujeres y las niñas y cuyo origen (explícito o no) está en la voluntad de control y sometimiento del género femenino existiendo un modelo socio-ideológico subyacente que determina los roles, actitudes e identidades de los sujetos sociales (hombres y mujeres) según su condición sexual y en la que influyen igualmente otras categorías como la clase, la raza, la etnia, la religión y la heteronormatividad, entre otras" (Zurbano-Berenguer, 2018, p. 83).

2. Se emplearán indistintamente los términos documentos deontológicos, códigos, recomendaciones, guías, manuales, 
protocolos y propuestas para referirnos al objeto de estudio, siendo conscientes de las diferencias existentes entre ellos (véase Aznar, 2005, p. 49-51).

3. También se ha incluido el Protocolo de la Federación Internacional de Periodistas (2008) porque, a pesar de no pertenecer únicamente al contexto español, entre sus miembros se encuentran destacadas asociaciones de periodistas y sindicatos españoles como la FAPE, FeSP, CCOO y ELA (Euskal Sindikatua).

4. Como ejemplo de buena praxis, cabe destacar la labor que viene realizando la Associació de Dones Periodistes de Catalunya, que desde 2009 analiza anualmente el impacto de las Recomendaciones sobre tratamiento de la violencia machista en los medios de comunicación (CAC, 2009) en los diarios de información general de ámbito nacional y autonómico.

\section{Referencias}

Aznar, Hugo (2005). Ética de la comunicación y nuevos retos sociales. Barcelona: Paidós Ibérica. ISBN: 8449316855

Bandrés, Elena (2011). “Propuesta para el tratamiento eficaz de la violencia de género". IC Revista científica de información y comunicación, n. 8, pp. 113-138.

https://idus.us.es/xmlui/handle/11441/33324

Carballido-González, Paula (2007). “Movimientos sociales y medios de comunicación: el cambio en el tratamiento de la violencia contra las mujeres". Recerca. Revista de pensament i anàlisi, n. 7, pp. 211-240.

http://www.e-revistes.uji.es/index.php/recerca/article/view/187/172

Carballido-González, Paula (2009). "Medios de comunicación social y violencia de género. Una revisión desde la teoría del framing". En: Bernardo-Paniagua, José-María; Martínez-García, Elena; Montiel-Roig, Gonzalo; Belando-Garín, Beatriz (eds.). Retos de la comunicación ante la violencia de género. Marco jurídico, discurso mediático y compromiso social. Valencia: Tirant lo Blanch, pp. 157-174. ISBN: 9788498766523

Cardoso, Ciro F. S. (2000). Introducción al trabajo de la investigación histórica. Conocimiento, método e historia. Barcelona: Crítica, 5a ed. ISBN: 8484320235

Comisión Europea (2016). Effectiveness of self- and co-regulation in the context of implementing the AVMS Directive. Netherlands: European Commission. ISBN: 9789279583933

https://doi.org/10.2759/035487

Dávila-Newman, Gladys (2006). "El razonamiento inductivo y deductivo dentro del proceso investigativo en ciencias experimentales y sociales". Laurus, v. 12, n. Ext., pp. 180-205.

http://www.redalyc.org/articulo.oa?id=76109911

Edo, Aurora (2017). "Estudio comparativo de los códigos deontológicos sobre violencia de género y comunicación. Bases para un manual de estilo fundamental". En: Suárez-Villegas, Juan-Carlos; Guadarrama-Rico, Luis-Alfonso; Valero-Vilchis, Jannet; Panarese, Paola (eds.). La desigualdad de género invisibilizada en la comunicación. Madrid: Dykinson, pp. 34-48. ISBN: 9788491481065

Fernández-Arribas, Javier; Noblejas, Myriam (2010). Cómo informar sobre violencia contra la mujer en las relaciones de pareja. Madrid: Centro Reina Sofía.

https://igualdade.xunta.gal/sites/default/files/files/documentos/como_informar_violencia_parella.pdf

Gallego-Ayala, Juana (2003). “De las recomendaciones a los mecanismos. Producción informativa y su incidencia en el tratamiento de la violencia de género". En: Ramírez-Alvarado, María-del-Mar (coord.). Medios de comunicación y violencia contra las mujeres. Sevilla: Instituto Andaluz de la Mujer, y Fundación Audiovisual de Andalucía, pp. 227-236. ISBN: 849326881 X

La marea (2015). “Propuestas para mejorar el tratamiento informativo de la violencia de género”. La marea, 24 noviembre. https://www.lamarea.com/2015/11/24/diagnostico-y-propuestas-sobre-el-tratamiento-informativo-de-la-violencia-de-genero

López-Díez, Pilar (dir.) (2006). Representación de la violencia de género en los informativos de TVE. Madrid: IORTV, e Instituto de la Mujer.

https://www.pilarlopezdiez.eu/pdf/RepreViolenciaTD.pdf

López-Díez, Pilar (2007). “La construcción de la violencia de género en los medios de comunicación”. En: Congreso abordaje integral de la violencia de género - actualización: Zamora, Auditorio del Campus Universitario Viriato 2-4 de mayo de 2007, pp. 34-48.

López-Díez, Pilar (2008). “Los medios y la representación de género: algunas propuestas para avanzar". Feminismo/s, n. 11, pp. 95-108.

https://doi.org/10.14198/fem.2008.11.06

Martínez-Rodríguez, Beatriz (2010). "El tratamiento informativo de la violencia contra la mujer en Galicia hoy: un análisis a la luz de los códigos deontológicos”. Observatorio (OBS) Journal, v. 4, n. 4, pp. 53-72.

http://obs.obercom.pt/index.php/obs/article/view/373 
Martínez-Rodríguez, Beatriz (2011). El tratamiento de la violencia contra la mujer a la luz de los códigos deontológicos. Tesis doctoral. Vigo: Universidad de Vigo.

http://www.investigo.biblioteca.uvigo.es/xmlui/handle/11093/454

Martínez-Rodríguez, Beatriz (2012). "Periodismo y responsabilidad social: el paradigma del tratamiento informativo de la violencia contra la mujer". Vivat academia, v. 14, n. 119, pp. 58-70.

https://doi.org/10.15178/va.2012.119.58-70

Martínez-Rodríguez, Beatriz (2013). “Vigencia y seguimiento de los códigos deontológicos monográficos sobre el tratamiento informativo de la violencia de género: el caso del diario Público". Ámbitos, n. 22.

http://institucional.us.es/ambitos/?p=85

Peris-Vidal, Manuel (2016). “La representación rigurosa del origen de la violencia machista en la prensa escrita: una propuesta de medición". Estudios sobre el mensaje periodístico, v. 22, n. 2, pp. 1123-1142. https://doi.org/10.5209/ESMP.54255

Rodríguez-Jiménez, Andrés; Pérez-Jacinto, Alipio-Omar (2017). “Métodos científicos de indagación y de construcción del conocimiento". Revista EAN, n. 82, pp. 179-200.

https://doi.org/10.21158/01208160.n82.2017.1647

Simons, Margaret; Khan, Elyas (2018). "Working with media to prevent violence against women - the uncovered intervention". Australian journalism review, v. 40, n. 1, pp. 51-63.

https://search.informit.com.au/documentSummary;dn=859308126055469;res=IELLCC

Soriano, Jaume (2004). “Las mujeres ante la información de violencia de género”. Sphera pública, n. 4, pp. 151-170. http://sphera.ucam.edu/index.php/sphera-01/article/view/15/24

Sutherland, Georgina; McCormack, Angus; Easteal, Patricia; Holland, Kate; Pirkis, Jane (2016). "Media guidelines for the responsible reporting of violence against women: A review of evidence and issues". Australian journalism review, v. 38 , n. 1, pp. 5-17.

https://ssrn.com/abstract $=2832441$

Sutherland, Georgina; McCormack, Angus; Pirkis, Jane; Easteal, Patricia; Holland, Kate; Vaughan, Cathyn (2015). Media representations of violence against women and their children: State of knowledge paper. Sydney: Anrows \& Our Watch. ISBN: 9781925372199

Valles, Miguel S. (1999). Técnicas cualitativas de investigación social. Reflexión metodológica y práctica profesional. Madrid: Síntesis. ISBN: 8477384495

https://metodologiaecs.files.wordpress.com/2014/11/vallesmiguel-tc3a9cnicas-cualitativas-de-investigacic3b3n-social-1999.pdf

Zurbano-Berenguer, Belén (2015a). "El tratamiento informativo y la responsabilidad ética en la información mediática de la violencia de género". En: $V$ Congreso para el estudio de la violencia contra las mujeres, pp. 46-56.

https://idus.us.es/xmlui/handle/11441/40074

Zurbano-Berenguer, Belén (2015b). Discurso periodístico y violencias contra las mujeres. Aproximaciones a la construcción y valoración de los mensajes informativos en la prensa de referencia en España (2000-2012). Tesis doctoral. Sevilla: Universidad de Sevilla.

https://idus.us.es/xmlui/handle/11441/27070

Zurbano-Berenguer, Belén (2018). “Comunicación, periodismo y violencias contra las mujeres en España: Reflexiones en torno a un estado de la cuestión". Revista observatorio, v. 4, n. 2, pp. 80-117.

https://doi.org/10.20873/uft.2447-4266.2018v4n2p80

Zurbano-Berenguer, Belén; Cano-Orón, Lorena; Liberia-Vayá, Irene (2018). “Gender studies in communication research: A longitudinal analysis of scientific papers published in Spanish journals indexed in the Journal Citation Reports (JCR) and the SCImago Journal Rank (SJR) (1988-2017)". Journal of research in gender studies, v. 8, n. 2, pp. 169-200.

https://doi.org/10.22381/JRGS82201810

Zurbano-Berenguer, Belén; García-Gordillo, María-del-Mar (2017). “Propuesta metodológica para la evaluación de la calidad deontológica de las noticias sobre violencias contra las mujeres". Communication \& society, v. 30, n. 1, pp. 73-85. https://www.unav.edu/publicaciones/revistas/index.php/communication-and-society/article/view/35796

Zurbano-Berenguer, Belén; Liberia-Vayá, Irene (2013). “Análisis crítico de los discursos audiovisuales sobre la violencia de género contra las mujeres: estudio comparado de caso". Estudios sobre el mensaje periodístico, v. 19, n. 2, pp. $921-939$. https://doi.org/10.5209/rev_ESMP.2013.v19.n2.43480

Zurbano-Berenguer, Belén; Martínez-Fábregas, Jezabel (2011). "Periodismo y violencia de género: la necesidad de un compromiso". En: Suárez-Villegas, Juan-Carlos (ed.). La ética de la comunicación a comienzos del siglo XXI. I Congreso internacional de ética de la comunicación. Sevilla: Eduforma, pp. 124-139. ISBN: 9788467658330

https://idus.us.es/xmlui/handle/11441/30975 


\section{Anexo 1. Documentos deontológicos sobre violencias contra las mujeres y medios de comunicación}

\begin{tabular}{|c|c|c|c|c|}
\hline Ref. & Año & Título & Autoría & Dónde \\
\hline 1 & 1999 & $\begin{array}{l}\text { Decálogo de recomendaciones a los medios de comunicación } \\
\text { para el tratamiento de la violencia contra las mujeres. Cómo } \\
\text { tratar bien los malos tratos }\end{array}$ & Instituto Andaluz de la Mujer & Andalucía \\
\hline 2 & 2000 & $\begin{array}{l}\text { Decálogo para los medios de comunicación sobre el tratamiento } \\
\text { de la violencia contra las mujeres }\end{array}$ & $\begin{array}{l}\text { Ayuntamiento de Pamplona, Universidad de } \\
\text { Navarra y Fundación IPES }\end{array}$ & Navarra \\
\hline 3 & 2001 & $\begin{array}{l}\text { Manual de estilo periodístico para informaciones sobre casos de } \\
\text { violencia doméstica o que afecten a menores }\end{array}$ & $\begin{array}{l}\text { Medios de comunicación de la Demarcación } \\
\text { de Girona }\end{array}$ & Cataluña \\
\hline 4 & 2002 & $\begin{array}{l}\text { Manual de urgencia sobre el tratamiento informativo de la } \\
\text { violencia contra las mujeres }\end{array}$ & IORTV e Instituto de la Mujer & España \\
\hline 5 & 2002 & $\begin{array}{l}\text { Notícies amb llaç blanc. Manual per a periodistes sobre la violèn- } \\
\text { cia domèstica }\end{array}$ & Unió de Periodistes Valencians & C. Valenciana \\
\hline 6 & 2003 & $\begin{array}{l}\text { Recomendaciones sobre el tratamiento de la violencia contra las } \\
\text { mujeres en los medios de difusión }\end{array}$ & Instituto Navarro de la Mujer & Navarra \\
\hline 7 & 2003 & $\begin{array}{l}\text { Protocolo para el tratamiento informativo de la violencia de } \\
\text { género y agresiones sexuales en La Rioja }\end{array}$ & Gobierno de La Rioja & La Rioja \\
\hline 8 & 2003 & $\begin{array}{l}\text { Decálogo para el tratamiento informativo de los malos tratos en } \\
\text { el entorno familiar }\end{array}$ & $\begin{array}{l}\text { Instituto Balear de la Mujer, Sindicato de Perio- } \\
\text { distas de las Islas Baleares y Gobierno de las Islas } \\
\text { Baleares }\end{array}$ & Islas Baleares \\
\hline 9 & 2004 & $\begin{array}{l}\text { Recomanacions del Consell de l'Audiovisual de Catalunya sobre } \\
\text { el tractament de la violència de gènere als programes informa- } \\
\text { tius i d'entreteniment als mitjans de comunicació }\end{array}$ & Consell de l'Audiovisual de Catalunya (CAC) & Cataluña \\
\hline 10 & 2004 & $\begin{array}{l}\text { Medios de comunicación e violencia de xénero. Declaración de } \\
\text { Compostela }\end{array}$ & $\begin{array}{l}\text { Observatorio Galego dos Medios y Colexio } \\
\text { Profesional de Xornalistas de Galicia }\end{array}$ & Galicia \\
\hline 11 & 2004 & $\begin{array}{l}\text { Decálogo básico (Decálogo de Zaragoza) para iniciar el debate } \\
\text { en los medios de comunicación }\end{array}$ & Ayuntamiento de Zaragoza & Aragón \\
\hline 12 & 2005 & $\begin{array}{l}\text { Tratamiento de la violencia contra las mujeres en los medios de } \\
\text { comunicación. Recomendaciones para las buenas prácticas en } \\
\text { la información sobre violencia de género }\end{array}$ & $\begin{array}{l}\text { Federación de Asociaciones de Periodistas } \\
\text { Españoles (FAPE) }\end{array}$ & España \\
\hline 13 & 2005 & $\begin{array}{l}\text { Decálogo sobre el tratamiento informativo de la violencia de } \\
\text { género en los medios de comunicación }\end{array}$ & $\begin{array}{l}\text { Agrupación de Mujeres Periodistas de la Asocia- } \\
\text { ción de la Prensa de Granada }\end{array}$ & Andalucía \\
\hline 14 & 2005 & $\begin{array}{l}\text { Decálogo. Recomendaciones para evitar una comunicación } \\
\text { sexista y androcéntrica y para el tratamiento adecuado de la } \\
\text { violencia de género }\end{array}$ & Consejo Comarcal de El Bierzo & Castilla y León \\
\hline 15 & 2005 & $\begin{array}{l}\text { Las noticias de los malos tratos. Propuestas para abordar la } \\
\text { información sobre la violencia de género }\end{array}$ & Instituto Aragonés de la Mujer & Aragón \\
\hline 16 & 2005 & $\begin{array}{l}\text { Lenguaje no sexista en medios de comunicación y publicidad. } \\
\text { Apuntes para la igualdad }\end{array}$ & Cabildo de Tenerife & Canarias \\
\hline 17 & 2006 & Código para el tratamiento informativo de la violencia de género & Junta de Castilla y León & Castilla y León \\
\hline 18 & 2007 & $\begin{array}{l}\text { Propuesta de decálogo para el tratamiento de la violencia de } \\
\text { género en los medios de comunicación }\end{array}$ & El Club de las 25 & España \\
\hline 19 & 2007 & $\begin{array}{l}\text { Protocolo de actuación periodística y publicitaria sobre igualdad } \\
\text { de oportunidades entre mujeres y hombres y tratamiento infor- } \\
\text { mativo sobre la violencia de género }\end{array}$ & Gobierno de Cantabria & Cantabria \\
\hline 20 & 2007 & $\begin{array}{l}\text { Medios de comunicación e violencia de xénero. Manual de } \\
\text { Redacción Xornalística }\end{array}$ & Xunta de Galicia & Galicia \\
\hline 21 & 2007 & $\begin{array}{l}\text { Decálogo de buenos usos en la información sobre violencia } \\
\text { doméstica }\end{array}$ & $\begin{array}{l}\text { Red Internacional de Mujeres Periodistas y } \\
\text { Comunicadoras de Cataluña }\end{array}$ & Cataluña \\
\hline 22 & 2008 & Decálogo para informar sobre violencia de género & Público & España \\
\hline 23 & 2008 & $\begin{array}{l}\text { La igualdad de género no es cuestión de suerte: Catálogo de } \\
\text { recomendaciones para el tratamiento de la violencia de género } \\
\text { en los medios de comunicación }\end{array}$ & Cabildo de Tenerife & Canarias \\
\hline 24 & 2008 & $\begin{array}{l}\text { Recomendaciones para un tratamiento informativo adecuado } \\
\text { de la violencia contra la mujer en los medios de comunicación }\end{array}$ & $\begin{array}{l}\text { I Congreso internacional mujer y medios de } \\
\text { comunicación (Cimmco) }\end{array}$ & C. Valenciana \\
\hline 25 & 2008 & $\begin{array}{l}\text { Protocolo de la FIP para la cobertura informativa de casos de } \\
\text { violencia contra las mujeres }\end{array}$ & Federación Internacional de Periodistas (FIP) & España \\
\hline 26 & 2009 & $\begin{array}{l}\text { Recomendaciones: El tratamiento de la violencia machista en los } \\
\text { medios de comunicación }\end{array}$ & Consell de l'Audiovisual de Catalunya (CAC) & Cataluña \\
\hline 27 & 2009 & $\begin{array}{l}\text { Los periodistas nos comprometemos. Manifiesto contra la } \\
\text { violencia de género }\end{array}$ & Asociación de la Prensa de La Rioja & La Rioja \\
\hline
\end{tabular}




\begin{tabular}{|c|c|c|c|c|}
\hline 28 & 2010 & $\begin{array}{l}\text { Recomendaciones genéricas de cómo informar sobre violencia } \\
\text { contra la mujer en las relaciones de pareja }\end{array}$ & Centro Reina Sofía & España \\
\hline 29 & 2010 & Intervención en publicidad y comunicación sexista & Instituto Canario de Igualdad & Canarias \\
\hline 30 & 2010 & $\begin{array}{l}\text { Código de los profesionales de CSTV para la elaboración de } \\
\text { informaciones sobre violencia machista }\end{array}$ & Canal Sur Televisión & Andalucía \\
\hline 31 & 2011 & $\begin{array}{l}\text { Recomendaciones: Tratamiento informativo para la igualdad de } \\
\text { género en Extremadura }\end{array}$ & $\begin{array}{l}\text { Instituto de la Mujer de Extremadura, Asociaciones } \\
\text { de la Prensa de Mérida, Cáceres y Badajoz y me- } \\
\text { dios de comunicación regionales extremeños }\end{array}$ & Extremadura \\
\hline 32 & 2011 & Diez pautas para un tratamiento eficaz de la violencia de género & Elena Bandrés Goldáraz & España \\
\hline 33 & 2013 & $\begin{array}{l}\text { Derecho a la información y justicia: Guía para el tratamiento } \\
\text { informativo de los procesos judiciales }\end{array}$ & Consejo Audiovisual de Andalucía (CAA) & Andalucía \\
\hline 34 & 2014 & $\begin{array}{l}\text { Informar sobre la violencia contra las mujeres. Guía de buenas } \\
\text { prácticas para los medios de comunicación }\end{array}$ & $\begin{array}{l}\text { Ayuntamiento de Zaragoza } \\
\text { (José María Calleja) }\end{array}$ & Aragón \\
\hline 35 & 2015 & $\begin{array}{l}\text { Decálogo. El papel garantista del Consejo Audiovisual de Anda- } \\
\text { lucía del tratamiento informativo ético de la violencia machista } \\
\text { en los medios }\end{array}$ & Ana Jorge Alonso & España \\
\hline 36 & 2015 & $\begin{array}{l}\text { Propuestas de la jornada: Tratamiento informativo de la violen- } \\
\text { cia machista en la era digital }\end{array}$ & $\begin{array}{l}\text { Participantes de la jornada organizada por } L a \\
\text { marea }\end{array}$ & Madrid \\
\hline 37 & 2016 & Guía para el tratamiento informativo de la violencia de género & Consejo Audiovisual de Andalucía (CAA) & Andalucía \\
\hline 38 & 2016 & $\begin{array}{l}\text { Protocolo de buenas prácticas: Tratamiento de la información } \\
\text { sobre violencia machista en los medios de comunicación }\end{array}$ & $\begin{array}{l}\text { Asociación de Periodistas de Aragón y Ayunta- } \\
\text { miento de Zaragoza }\end{array}$ & Aragón \\
\hline 39 & 2016 & $\begin{array}{l}\text { Decálogo para el tratamiento informativo de la violencia contra } \\
\text { las mujeres }\end{array}$ & $\begin{array}{l}\text { Begira (Comisión Asesora para un uso no sexista } \\
\text { de la publicidad y la comunicación) } \\
\text { Emakunde (Instituto Vasco de la Mujer) }\end{array}$ & País Vasco \\
\hline 40 & 2017 & Periodismo contra las violencias machistas & La marea y Oxfam Intermón & España \\
\hline 41 & 2017 & Guía para el tratamiento informativo de la violencia de género & Junta de Castilla y León & Castilla y León \\
\hline 42 & 2018 & $\begin{array}{l}\text { Decálogo para el tratamiento periodístico de las víctimas de la } \\
\text { violencia machista }\end{array}$ & Asociación de Periodistas por la lgualdad & Aragón \\
\hline 43 & 2018 & $\begin{array}{l}\text { Manual d'estil per al tractament de la violència masclista i el } \\
\text { llenguatge inclusiu en els mitjans de comunicació }\end{array}$ & Unió de Periodistes Valencians & C. Valenciana \\
\hline 44 & 2007 & $\begin{array}{l}\text { La violencia hacia las mujeres en los medios de comunicación. } \\
\text { Transformando las noticias }\end{array}$ & Ciscsa y Red Mujer y Hábitat de América Latina & Argentina \\
\hline 45 & 2008 & $\begin{array}{l}\text { Decálogo para el tratamiento periodístico de la violencia contra } \\
\text { las mujeres }\end{array}$ & $\begin{array}{l}\text { PAR (Periodistas de Argentina en Red por una } \\
\text { comunicación no sexista) }\end{array}$ & Argentina \\
\hline 46 & 2008 & $\begin{array}{l}\text { Lentes lilas para los medios de comunicación. La construcción de } \\
\text { las noticias sobre violencia hacia las mujeres }\end{array}$ & $\begin{array}{l}\text { UNFPA y Secretaría de la Mujer. Presidencia de la } \\
\text { República del Paraguay }\end{array}$ & Paraguay \\
\hline 47 & 2009 & $\begin{array}{l}\text { Manual de género para periodistas: recomendaciones básicas } \\
\text { para el ejercicio del periodismo con enfoque de género }\end{array}$ & $\begin{array}{l}\text { América Latina Genera (Área Práctica de Género } \\
\text { Rsclac PNUD) }\end{array}$ & $\begin{array}{l}\text { América } \\
\text { Latina }\end{array}$ \\
\hline 48 & 2009 & $\begin{array}{l}\text { Noticias que salvan vidas. Manual periodístico para el abordaje } \\
\text { de la violencia contra las mujeres }\end{array}$ & $\begin{array}{l}\text { Amnistía Internacional Argentina - Asociación } \\
\text { Civil Pro Amnistía }\end{array}$ & Argentina \\
\hline 49 & 2010 & $\begin{array}{l}\text { Por un periodismo no sexista. Pautas para comunicar desde una } \\
\text { perspectiva de género en Chile }\end{array}$ & Orealc/Unesco, Santiago & Chile \\
\hline 50 & 2011 & $\begin{array}{l}\text { La violencia tiene mil caras. Guía para profesionales y comuni- } \\
\text { cadores/as de medios de comunicación en violencia contra las } \\
\text { mujeres }\end{array}$ & UNFPA & Chile \\
\hline 51 & 2011 & $\begin{array}{l}\text { Pautas para el tratamiento informativo adecuado de la violencia } \\
\text { contra la mujer en los medios de comunicación }\end{array}$ & Ministerio de la Mujer y Desarrollo Social & Perú \\
\hline 52 & 2011 & $\begin{array}{l}\text { Proyecto de ley para el tratamiento mediático de la violencia de } \\
\text { género }\end{array}$ & Gustavo Ferrari & Argentina \\
\hline 53 & 2011 & $\begin{array}{l}\text { Manual para periodistas y comunicadores sobre el tratamiento } \\
\text { de noticias de violencia familiar }\end{array}$ & $\begin{array}{l}\text { Acción por los Niños y Centro de Capacitación } \\
\text { J.M. Arguedianos }\end{array}$ & Perú \\
\hline 54 & 2014 & $\begin{array}{l}\text { Violencias puertas adentro. Guía para una cobertura periodística } \\
\text { con enfoque de género y generaciones }\end{array}$ & $\begin{array}{l}\text { Consejo Nacional Consultivo de Lucha contra } \\
\text { la Violencia Doméstica y Sistema Integral de } \\
\text { Protección a la Infancia y Adolescencia contra } \\
\text { la Violencia }\end{array}$ & Uruguay \\
\hline 55 & 2014 & $\begin{array}{l}\text { ¿Los periodistas podemos ayudar? Manual para periodistas que } \\
\text { informan sobre violencia de género }\end{array}$ & Conexión - Fondo de emancipación & Bolivia \\
\hline 56 & 2015 & $\begin{array}{l}\text { Periodismo libre de violencia. Protocolo para el abordaje infor- } \\
\text { mativo de la violencia contra las mujeres basada en género }\end{array}$ & $\begin{array}{l}\text { Ministerio de Comunicación del Estado Plurina- } \\
\text { cional de Bolivia }\end{array}$ & Bolivia \\
\hline 57 & 2016 & $\begin{array}{l}\text { Guía para el tratamiento mediático responsable de casos de } \\
\text { violencia contra las mujeres }\end{array}$ & $\begin{array}{l}\text { Defensoría del Público de Servicios de Comuni- } \\
\text { cación Audiovisual }\end{array}$ & Argentina \\
\hline 58 & 2017 & ¿Cómo abordar noticias de violencia contra las mujeres? & Ministerio de la Mujer y Poblaciones Vulnerables & Perú \\
\hline
\end{tabular}

